\title{
COVID-19 and Orthopaedics: Recovery After the Pandemic Surge
}

\author{
Brandon Petrone, D.O., Cesar R. Iturriaga, D.O., Thomas Mauri, M.D., and \\ Nicholas Sgaglione, M.D.
}

\begin{abstract}
The novel Coronavirus (COVID-19) pandemic has placed an immense strain on health care systems and orthopedic surgeons across the world. To limit the spread, federal and state governments mandated the cancellation of all nonurgent surgical cases to address surging hospital admissions and manage workforce and resource reallocation. During the pandemic surge, thousands of surgical cancellations have been required. We outline our experience through the onset and advance of the surge, detail our incident response and discuss the transition toward recovery. Level of Evidence: Level V
\end{abstract}

$\mathbf{S}^{\mathrm{c}}$ evere acute respiratory syndrome coronavirus-2 (SARS-CoV-2), the virus responsible for coronavirus disease-19 (COVID-19), is the most impactful health care challenge of our generation. Originating in China in December 2019, the novel coronavirus was declared a pandemic by the World Health Organization on March 11, 2020. ${ }^{1}$ On March 16, the Imperial College COVID Response Team projection models estimated that without containment, interventions the United States could see a peak death toll of 2.2 million people by September $2020 .^{2}$

In the United States, on a regional or statewide basis, drastic restrictions to mitigate the spread of the virus have been implemented, with more than $90 \%$ of Americans affected by a stay-at-home order. Policies have limited travel, mandated social distancing and protective masks and closed nonessential businesses. Health care institutions have likewise taken measures in anticipation of a surge of infected patients. ${ }^{3}$

From Department of Orthopaedics, Northwell Health, Great Neck, New York, U.S.A.

The authors report that they have no conflicts of interest in the authorship and publication of this article. Full ICMJE author disclosure forms are available for this article online, as supplementary material.

Received May 12, 2020; accepted May 18, 2020.

Corresponding Author: Nicholas Sgaglione, M.D., Department of Orthopaedic Surgery, Northwell Health, 611 Northern Boulevard, Great Neck, New York 11021, U.S.A.E-mail: nsgagli@northwell.edu

Published by Elsevier on behalf of the Arthroscopy Association of North America. This is an open access article under the CC BY-NC-ND license (http://creativecommons.org/licenses/by-nc-nd/4.0/).

2666-061X/20841

https://doi.org/10.1016/j.asmr.2020.05.009
In New York (NY) state, the first confirmed COVID-19 case occurred on March 1, 2020, and exponential spread of the virus across the NY City metropolitan area resulted in a global epicenter. The first COVID-19 patient was admitted to a Northwell Health hospital on March 4, 2020. The Northwell Health System is the largest health care system in NY state, with 23 total hospitals, 5,692 beds, 18 office sites, and 14 ambulatory surgery center (ASC) sites carrying out more than 33,000 orthopedic surgical cases yearly. A surge of infected patients followed, culminating in a peak of cases requiring admissions and complex resuscitative treatment.

Crisis-management teams assembled to prepare for reorganization of hospital and ambulatory operations, including a large-scale contraction of orthopedic operations. This resulted in the reduction of ambulatory patient visits and the postponement of thousands of surgeries. Efforts to expand the COVID-19 treatment teams additionally prompted the redeployment of $14 \%$ of the orthopedic attending faculty staff, $40 \%$ of residents and fellows and $82 \%$ of advanced clinical practitioners, including physician assistants and nurse practitioners.

Our purpose is to describe our experience through the onset and advance of the surge, detail our incident response and discuss the transition toward recovery.

\section{Onset of the Surge}

As the largest health care provider in the state, Northwell Health was on the frontlines during the response to the outbreak. Devastating consequences on 
the healthcare system were witnessed as the proportion of daily hospital admissions from the EDs exploded from $20 \%$ to over $45 \%$ at the peak in early April. More than 6,000 patients with suspected COVID-19 were admitted across the health system, with $28 \%$ requiring admissions to the intensive care units and $25 \%$ requiring mechanical ventilators. Our Emergency Operations Center saw the early need for personnel and resource reallocation at the 5 tertiary and 11 community hospitals in which orthopedic surgery is performed in our health system. By early April, institutional hospital capacity had increased by $23 \%$, effectively creating 1,200 additional beds above the pre-existing capacity of 5,200 . To "load-balance" the patient surge across the system, patients in intensive care units were transferred from overcrowded smaller hospitals to larger tertiary centers.

\section{Incident Response}

\section{Safety Strategies}

Protecting the health care team was pivotal in the face of unclear viral transmission, disease expression and serologic immunity phenomena. Important infectionmitigation strategies included adequately supplying and conserving limited supplies of personal protective equipment (PPE) as well as providing exposure training to providers and patients. Distribution of personal protective equipment was prioritized to the front-line providers and testing centers. ${ }^{4,5}$

\section{Testing}

Initially, testing was sorely limited, and false-negative controls plagued testing efforts. ${ }^{6}$ Gradually, new technologies improved testing sensitivity, specificity and availability. ${ }^{7}$ This allowed us to successfully test almost all patients and at-risk health care workers, inspiring confidence in our staff and bolstering effective patient care.

\section{Elective-Surgical Cancellation}

During the onset, Northwell Health mandated the cancellation of all nonurgent surgical cases to address surging admissions and workforce and resource reallocation. Only emergent orthopaedic cases continued. ${ }^{8}$ Operative patients were admitted directly to the hospital, avoiding exposure in the ED. Encouragement of timely surgery and fast-tracking of medical clearances, radiologic studies and physical therapy minimized inpatient overnight stays and helped to expedite discharges. Our health care system performed approximately 2,970 emergent surgeries during a 4-week period at our 16 orthopedic hospital sites, efficiently utilizing limited hospital resources while providing imperative patient care.

Emergent orthopaedic surgical cases continued to be performed on the basis of clinical criteria, including acute fractures, dislocations, tendon and ligament ruptures, and any cases in which a delay would likely result in an adverse result ${ }^{8}$ (Fig 1). Postponed cases (orthopaedic and nonorthopaedic) were tracked and entered into a database using the American College of Surgeons case acuity triage guidelines. ${ }^{9}$ Nonsurgical treatments for certain conditions were carefully adopted in selected cases as long as the timing of the procedure would not affect clinical outcomes. Finally, the process for rescheduling these postponed cases is expanded on below.

\section{Redeployment and Reorganization}

As above, $14 \%$ of orthopedic attending faculty staff, $40 \%$ of residents and fellows and $82 \%$ of advanced clinical practitioners, including physician assistants and nurse practitioners, were redeployed to COVID-19 treatment teams to manage both the surge of patients with COVID19 and to replace other providers who were quarantined for potential viral exposure or became ill with COVID-19. An "all hands on deck" attitude prevailed.

To alleviate workloads in the EDs, a musculoskeletal fast-track was created, streamlining all patients with musculoskeletal issues to orthopedic providers for evaluation, while avoiding the ED as much as possible. However, with stay-at-home orders in place, a decline in acute traumatic injuries led to diminished need for orthopaedic care.

\section{Leadership and Emotional Support}

Particularly because of the virulence of the virus, as well as the uncertainty resulting from this new pathogen, health care workers were at risk of contracting the virus and transmitting the virus to their patients and families. At-risk health care workers require clear communication, emotional support and effective leadership. Despite resilience, uncertainty, fear and loss have been physically and psychologically exhausting. Social and familial disruption, unfamiliar settings, exhausting shift work, and a near $88 \%$ death rate in ventilated patients ${ }^{10}$ have affected the health care team's mental health. Evidence from this pandemic and prior crises has demonstrated the importance of maintaining health care workers' wellness. ${ }^{8,11}$ Along with mitigation strategies, Northwell Health has comprehensively supported our health care workers by providing educational classes, mental health resources and grief counseling. Protecting the health of our frontline providers has been a foremost concern.

\section{Telehealth Outpatient Services}

The use of telehealth services allows us to provide health care while minimizing the risk of disease transmission. ${ }^{12,13}$ Telehealth capabilities were already integrated into certain specialties prior to the pandemic, most notably in behavioral health services in the ED. The rapid adoption and expansion of telehealth services 
Fig 1. Classification of priority level when considering operative procedures after restarting elective surgery.
- Delay would cause immediate severe harm or death

-Delay would result in permanent disability or severe harm

- Patient is medically unstable related to a surgical condition

- Delay would prolong current hospital stay or increase the likelihood of later hospital admission during the pandemic

- Patients have failed medical management of a surgical condition

Urgent

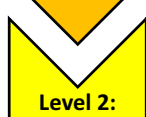

- Delay or further delay beyond 3 months risks patient harm

- Delay would increase the risk of a hospital stay or create the likelihood of later hospital admission

Semi-Urgent

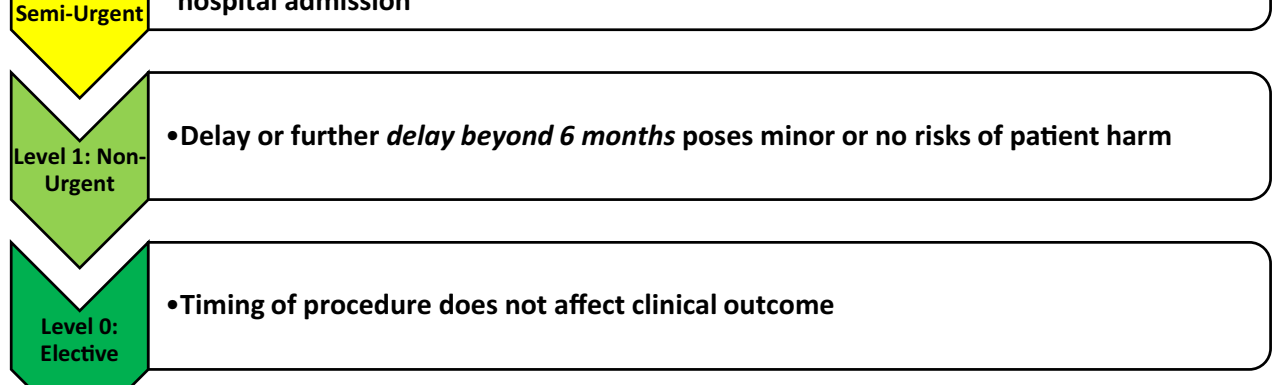

by our orthopaedic department was key in engaging actively symptomatic and postoperative patients and instrumental in maintaining continuity of care.

\section{Education}

Remote-access technology has been invaluable in orthopaedic resident education, and rapid implementation allowed our program to continue the scheduled curriculum. Virtual platforms have become common in residency training during the pandemic, ${ }^{14}$ facilitating remote sharing of education resources among orthopaedic residents and attending faculty. Cancellation of elective surgeries, interhospital residency rotations and in-person skills laboratories may have repercussions on resident training and surgical proficiency. Although the Accreditation Council for Graduate Medical Education has suspended minimum case requirements for competency, ${ }^{15}$ our residency programs are exploring innovative ways to practice surgical skills through weekly training modules, online webinars and remote-access virtual reality proficiency-based training.

\section{Transition Toward Recovery}

In April, the White House released guidelines on a stepwise approach to reopening the country. ${ }^{16}$ Regions need to document downward trends in suspected and confirmed COVID-19 cases over a 14-day period, safely restore noncrisis inpatient and critical-care bed capacities and provide a testing program for repatriated health care staff. Regions that satisfy this gating criteria may enter into phase 1, allowing for elective outpatient surgery to be reinstated at COVID-contained facilities.
States not demonstrating rebound infections, while meeting the gating criteria after implementation of phase 1, may progress into phase 2 and resume elective surgeries on outpatient and inpatient bases. States with no evidence of rebound infections, while continuing to meet the gating criteria after implementing phase 2 , may progress to phase 3 . This lifts visitation restrictions in hospitals and senior care facilities. ${ }^{16}$

\section{Patient and Provider Safety}

Restoration of elective orthopaedic procedures requires downward trends in active cases, widespread disease and serologic testing and effective contact tracing. Performing elective procedures without creating a safe hospital environment can have adverse effects on outcomes and may lead to resurgence. Patients undergoing elective orthopaedic surgery who were treated while the patient was in the incubation period of COVID-19 infection resulted in a $44.1 \%$ rate of admission to intensive care units postoperatively, with a mortality rate of $20.5 \% .{ }^{17}$ Poor surgical outcomes in patients with undiagnosed COVID-19, along with a $25 \%-50 \%$ transmission rate from asymptomatic carriers, necessitates testing in order to limit the potential spread of disease burden. ${ }^{18}$

Initiation of preoperative testing protocols can be an effective screening method for protecting against exposure and resurgence. ${ }^{19}$ Patients log their daily temperatures on days before surgical admission and are screened for any symptoms on arrival. All patients should be tested 24-48 hours prior to their procedures, with repeat testing if the clinician has high index of 


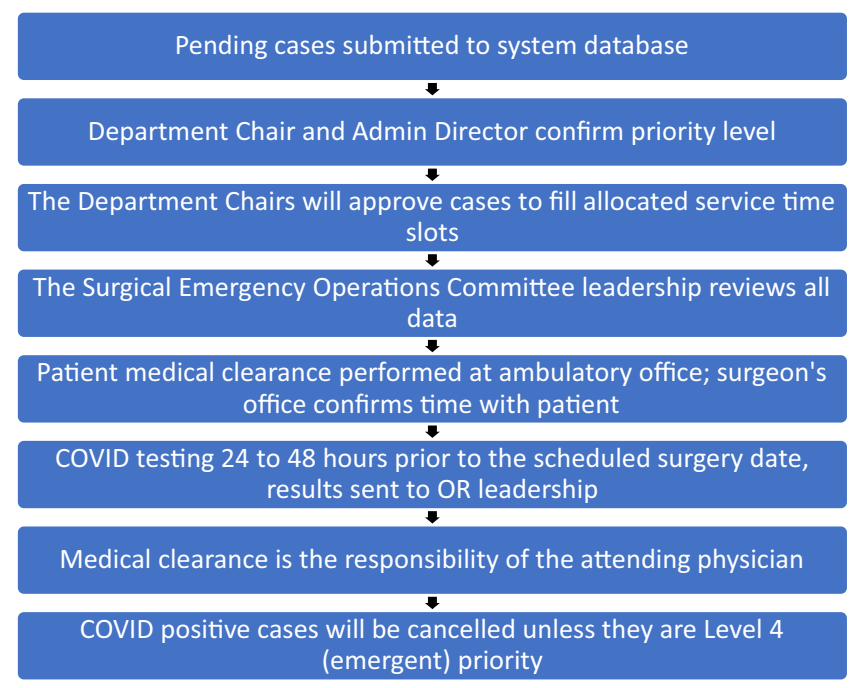

Fig 2. Process for operating room case scheduling for identified postponed cases.

suspicion in the presence of a negative test result. Additionally, patients who develop new symptoms consistent with a newly acquired infection or worsening symptoms after previously testing negative or inconclusive for COVID-19 must be tested again. Finally, those patients previously testing positive must successfully complete a 2 -week quarantine period and then be retested before their surgeries.

Commitment to the safety of our patients, clinicians and team members is our utmost priority, and we have implemented many processes to ensure that this commitment is honored. They include the creation of dedicated, segregated operating rooms (ORs), postanesthesia care unit nurses and inpatient units for all levels of care for non-COVID surgery. To maintain a safe environment within the hospital, Northwell Health has begun surface and air SARS-CoV-2 contamination sampling. A quantitative viral heat map is generated, directing decontamination and safety protocols in high viral-load areas. Furthermore, Northwell Health will reopen and staff dedicated COVID-19-contained ASCs and inpatient hospitals for elective procedures.

Wearable sensor technology can track trends in resting heart rate and daily activity to identify seasonal respiratory illnesses ${ }^{20}$ and may serve to detect and contain outbreaks. Additionally, contact tracing has been a pillar of disease control in public health, ${ }^{21}$ and implementation of exposure-alert systems are being facilitated by partnerships between the Centers for Disease Control and Prevention and software companies. As we reinstate elective surgery, these tracing methods foster a safe environment by monitoring active cases.

\section{Restarting the OR-Logistical Challenges}

As health care institutions more effectively manage SARS-CoV-2, consideration must be given to the logistics of reinstituting elective orthopaedic procedures. Multiple factors need to be addressed, including staff reintegration and workflow, elective surgical rescheduling and prioritization guidelines, OR block time distribution, preferential use of ASCs, and preoperative protocols.

\section{Staff Reintegration}

As the demand for OR time escalates, so will the need for OR and perioperative staff to return from redeployment in COVID-positive units. Repatriation of perioperative and OR staff will allow for restoration of orthopaedic OR and postanesthesia teams. To avoid deleterious effects on patient care, the transition must be gradual, ensuring adequate coverage of remaining critical care patients by using teamwork and extensive communication among all health professionals. ${ }^{22}$ Once front-line subspecialties no longer need extra assistance, and removing staff will not disrupt patient care, redeployed personnel will be reintegrated back to their primary positions. Before restarting, employees will be given the opportunity to obtain, voluntarily, antibody testing. Employees with high-risk exposure to COVID-19 will be allowed 2 weeks to quarantine and will undergo repeat testing before returning. Once capacity, workforce and safety issues have been addressed, elective procedures can begin.

\section{Approach to Operative Case Priority}

Redistribution of OR availability, a precious commodity, must be handled in an ethical, judicious and patient-centric manner, which will be handled by the Surgical Emergency Operations Committee (SEOC). Considering that more than 16,000 postponed surgical cases, including 1,800 orthopedic cases, need to be performed at our institution, the SEOC will use clinical guidelines and a multidisciplinary approach to prioritization based on the exigency and severity of the patient's condition, fostering transparent, equitable and ethical decisions. ${ }^{8,23}$ Cases will be classified into priority levels using time-sensitive criteria corresponding to their likelihood of harm due to delays in surgery, as outlined in Fig 1. It is the surgeons' responsibility to

\footnotetext{
Segregated operating rooms, recovery units, and inpatient units have been created to exclusively treat non-COVID positive patients

The Endoscopy unit operates as a non-COVID facility and does not currently treat COVID positive patients

All patients are screened for COVID-19 prior to entry into the preoperative areas and tested before admission or any procedure.

Ample personal protective equipment is available for both patients and healthcare workers
}

Fig 3. Talking points to highlight safety measures and inspire patients' confidence. 
identify initially the level 4 (emergent) and level 3 (urgent) priority cases that have been postponed. They will submit case information via the system's database to the department chair of each service for approval prior to scheduling (Fig 2). The Surgical Emergency Operations Committee will provide oversight of all scheduled cases. Once approved, department administrators will contact the surgeon to provide booking information. After the case is booked, presurgical and COVID-19 polymerase chain reaction testing will be the responsibility of the attending surgeon and team. Cases without a resulting COVID-19 polymerase chain reaction test will be cancelled to ensure the safety of all involved (Fig 2). In regard to level 2 (semiurgent) and level 1 (nonurgent) cases, as well as elective orthopaedic surgery cases, a phased-in approach is anticipated and is being prepared for on the basis of NY state regulatory approval. The beginning of elective surgical cases is predicated, in our state, by reduced disease burden and hospital capacity as outlined by the phase 3 criteria provided by the White House. ${ }^{16}$

\section{Novel Adaptations}

The potential implementation of 7-day-a-week elective surgery scheduling will allow for improved volume, but workforce issues will have to be addressed. This workflow initiative is being modeled by the hiring of new OR staff, staggering existing OR team shifts, promoting positive leadership, and adding necessary resources and employee incentives. ${ }^{24}$ Consideration will be given to directing outpatient procedures to ASCs, while directing hospital procedures to those requiring admission for $>23$ hours, further maximizing non-COVID-19 patient safety and hospital bed and OR availability. ${ }^{8,25}$ Additional initiatives have begun to expand traditional ASC-block schedules to incorporate limited-stay cases to adopting 23-hour postrecovery schedules.

\section{Patients' Experiences}

This pandemic has had a significant effect on the hospital system, but we must also be cognizant of the impacts on patients. With high pandemic-related unemployment, some patients may struggle to afford surgery. ${ }^{26,27}$ During the 2008 global recession, financially motivated cancellations of elective orthopaedic cases increased as the economic situation worsened, correlating positively with the unemployment rate and negatively with job availability. ${ }^{27}$ In addition, economic downturns negatively affect health metrics: physician visits, diagnostic rates, surgical procedures, and disease incidence. ${ }^{28,29}$ Surgical postponement can have deleterious effects on patients' mental and physical well-being. Health initiatives must focus on supportive patient communication. Regaining patients' confidence by letting them know that seeking elective health care is safe, as well as accurately informing them of risks, including COVID-19 transmission risks, could begin in the outpatient setting. Providers must understand that patients may have concerns about undergoing surgery at this time or about their potential exposure to COVID-19 in doing so. Regardless of the risks, patients must be well informed; a trusting physicianpatient relationship is instrumental in providing highquality care and fostering patient satisfaction. Northwell Health has recommended key talking points that surgeons can share with their patients; they are outlined in Fig 3. Continued use of telemedicine may minimize patient exposure during physical therapy and rehabilitation and may be used pre- and postoperatively. ${ }^{12}$ Implementation of digital technology will support clinical care, including patient rehabilitation during the recovery phase.

\section{Conclusions}

The ramifications of this pandemic and the anticipated radical changes that it may cause in our health care system and our society are still not fully understood. The future remains unclear but it is obvious that the scope of our practice has been dramatically altered. $^{30,31}$ Orthopaedic surgery and health care in general will evolve rapidly, and changes will be incorporated into our standards of care. Evidence-based protocols and innovative technology enable efficient identification of risk, resource use and minimization of exposure. ${ }^{31}$

The challenges we face are shared in the orthopaedic community and should serve to galvanize our efforts. As we progress through this ordeal, preparations must be made to transition our health care system safely from disaster operations back to conventional patient care. The SARS-CoV-2 pandemic is a test of global cooperation; only through dissemination of knowledge can we overcome this existential threat.

\section{References}

1. World Health Organization Director-General's. Opening Remarks at the Media Briefing on COVID-19. March 11, 2020. Available at: www.who.int/dg/speeches/detail/whodirector-general-s-opening-remarks-at-the-media-briefingon-covid-19-11-march-2020. Accessed April 18, 2020.

2. Ferguson NM, Laydon D, Nedjati-Gilani G, et al. Impact of non-pharmaceutical interventions (NPIs) to reduce COVID-19 mortality and health care demand. London, UK: Imperial College COVID-19 Response Team, March 16, 2020.

3. Coronavirus. New York City hospitals hope they "have hit a plateau." Available at: https://www.usatoday.com/story/ news/health/2020/04/16/coronavirus-new-york-cityhospitals-plateau/299225800 1/. Accessed May 16, 2020.

4. Guidance on Preparing Workplaces for COVID-19. Available at: https://Www.osha.gov/Publications/OSHA3990. pdf. Accessed April 19, 2020. 
5. Protecting Health care Personnel | HAI | CDC. Available at: https://www.osha.gov/Publications/osha3151.pdf. Accessed April 19, 2020.

6. Patel NV. Why the CDC botched its coronavirus testing. MIT Technol Rev, 2020. Available at, www.technologyreview. com/2020/03/05/905484/why-the-cdc-botched-its-corona virus-testing/. Accessed April 18, 2020.

7. Centers for Disease Control and Prevention. Information for laboratories: 2019-nCoV. Available at: https://www. cdc.gov/coronavirus/2019-ncov/lab/index.html. Accessed April 22, 2020.

8. Massey PA, McClary K, Zhang AS, et al. Orthopaedic surgical selection and inpatient paradigms during the coronavirus COVID-19 pandemic. J Am Acad Orthopaed Surg 2020;28:436-450.

9. American College of Surgeons. COVID-19: Recommendations for management of elective surgical procedures. Mar 13. Available at: https://www.facs.org/covid-19/clinicalguidance/elective-surgery 2020. Accessed April 17, 2020.

10. Richardson S, Hirsch JS, Narasimhan M, et al. Presenting characteristics, comorbidities, and outcomes among 5700 patients hospitalized with COVID-19 in the New York City area. JAMA. Published online April 22, 2020.

11. Guo X, Wang J, Hu D, et al. Survey of COVID-19 disease among orthopaedic surgeons in Wuhan, People's Republic of China. J Bone Joint Surg 2020;102:847-854.

12. Parisien R, Shin M, Constant M, et al. Telehealth utilization in response to the novel coronavirus (COVID-19) pandemic in orthopaedic surgery. J Am Acad Orthopaed Surg 2020. Volume published ahead of print.

13. Constanzo F, Aracena-Sherck P, Hidalgo JP, et al. Contribution of a synchronic teleneurology program to decrease the patient number waiting for a first consultation and their waiting time in Chile. BMC Med Informat Decis Mak 2020;20:20.

14. Kogan M, Klein S, Hannon C, Nolte M. Orthopaedic education during the COVID-19 pandemic. J Am Acad Orthopaed Surg 2020. Volume published ahead of print.

15. Accreditation Council for Graduate Medical Education response to the Coronavirus (COVID-19). Mar 18, 2020. Available at: https://acgme.org/Newsroom/NewsroomDetails/ArticleID/10111/ACGME-Response-to-the-Corona virus-COVID-19. Accessed April 20, 2020.

16. Guidelines for Opening Up America Again. Available at: https://www.whitehouse.gov/openingamerica/. Accessed April 16, 2020.

17. Lei S, Jiang F, Su W, et al. Clinical characteristics and outcomes of patients undergoing surgeries during the incubation period of COVID-19 infection. E ClinMed 2020;21:100331.

18. Bai Y, Yao L, Wei T, et al. Presumed asymptomatic carrier transmission of COVID-19. JAMA 2020;323:1406-1407.

19. Liang ZC, Wang W, Murphy D, et al. Novel Coronavirus and orthopaedic surgery. J Bone Joint Surg 2020;102:745-749.

20. Radin JM, Wineinger NE, Topol EJ, Steinhubl SR. Harnessing wearable device data to improve state-level realtime surveillance of influenza-like illness in the USA: A population-based study. Lancet Digital Health 2020;2: 65-93.

21. Scutchfield F, Douglas X. Principles of public health practice. Clifton Park, New York, USA: Delmar Learning. 2003. p. 71. ISBN 0-76682843-3.

22. Babiker A, El Husseini M, Al Nemri A. Health care professional development: Working as a team to improve patient care. Sudan J Paediatr 2014;14:9-16.

23. Centers for Medicare and Medicaid Services. Non-Emergent, Elective Medical Services, and Treatment Recommendations: https://Www.cms.gov/files/document/ 31820-cms-adult-elective-surgery-and-procedures-recommendations.pdf. Accessed April 17, 2020.

24. Sillero A, Adelaida Z. Organizational factors and burnout of perioperative nurses. Clin Prac Epidemiol Ment Health 2018;14:132-142.

25. Crawford DC, Li CS, Sprague S, Bhandari M. Clinical and cost implications of inpatient versus outpatient orthopedic surgeries: A systematic review of the published literature. Orthoped Rev 2015;7:6177.

26. Wolfers J. The unemployment rate is probably around 13 percent. Apr 3, 2020. Available at: https://www.nytimes. com/2020/04/03/upshot/coronavirus-jobless-rate-greatdepression.html. Accessed April 20, 2020.

27. Klein GR, Parcells BW, Levine HB, et al. The economic recession and its effect on utilization of elective total joint arthroplasty. Am J Orthoped 2013;42:499-504.

28. Fujihara N, Lark ME, Fujihara Y, Chung KC. The effect of economic downturn on the volume of surgical procedures: A systematic review. Int J Surg 2017;44:56-63.

29. Iorio R, Davis Cm III, Healy WL, Fehring TK, et al. Impact of the economic downturn on adult reconstruction surgery: A survey of the American Association of Hip and Knee Surgeons. J Arthroplast 2010;25:1005-1014.

30. Vaccaro A, Getz C, Cohen B, et al. Practice management during the COVID-19 pandemic. J Am Acad Orthopaed Surg 2020;28:464-470.

31. Anoushiravani A, O'Connor C, DiCaprio M, Iorio R. Economic impacts of the COVID19 crisis: An orthopaedic perspective. JBJS Open Access 2020;102:937-941. 